\title{
BMJ Open Comparison of FORTA, PRISCUS and EU(7)-PIM lists on identifying potentially inappropriate medication and its impact on cognitive function in multimorbid elderly German people in primary care: a multicentre observational study
}

\author{
Caroline Krüger (D) , ${ }^{1}$ Ingmar Schäfer (D) , ${ }^{2}$ Hendrik van den Bussche, ${ }^{2}$ \\ Horst Bickel, ${ }^{3}$ Tobias Dreischulte, ${ }^{4,5}$ Angela Fuchs, ${ }^{6}$ Hans-Helmut König, ${ }^{7}$ \\ Wolfgang Maier, ${ }^{8}$ Karola Mergenthal, ${ }^{9}$ Steffi G Riedel-Heller, ${ }^{10}$ Gerhard Schön, ${ }^{11}$ \\ Siegfried Weyerer, ${ }^{12}$ Birgitt Wiese, ${ }^{13}$ Wolfgang von Renteln-Kruse, ${ }^{14}$ \\ Claudia Langebrake, ${ }^{1,15}$ Martin Scherer ${ }^{2}$
}

To cite: Krüger C, Schäfer I, van den Bussche $\mathrm{H}$, et al. Comparison of FORTA, PRISCUS and EU(7)-PIM lists on identifying potentially inappropriate medication and its impact on cognitive function in multimorbid elderly German people in primary care: a multicentre observational study. BMJ Open 2021;11:e050344. doi:10.1136/ bmjopen-2021-050344

- Prepublication history and additional supplemental material for this paper are available online. To view these files, please visit the journal online (http://dx.doi.org/10.1136/ bmjopen-2021-050344).

Received 17 February 2021 Accepted 31 August 2021

Check for updates

(c) Author(s) (or their employer(s)) 2021. Re-use permitted under CC BY-NC. No commercial re-use. See rights and permissions. Published by BMJ.

For numbered affiliations see end of article.

Correspondence to Mrs Caroline Krüger; c.krueger@uke.de

\section{ABSTRACT}

Objectives Our study aimed to assess the frequency of potentially inappropriate medication (PIM) use (according to three PIM lists) and to examine the association between PIM use and cognitive function among participants in the MultiCare cohort.

Design MultiCare is conducted as a longitudinal, multicentre, observational cohort study.

Setting The MultiCare study is located in eight different study centres in Germany.

Participants 3189 patients (59.3\% female).

Primary and secondary outcome measures The study had a cross-sectional design using baseline data from the German MultiCare study. Prescribed and over-the-counter drugs were classified using FORTA (Fit fOR The Aged), PRISCUS (Latin for 'time-honoured') and EU(7)-PIM lists. A mixed-effect multivariate linear regression was performed to calculate the association between PIM use patients' cognitive function (measured with (LDST)).

Results Patients (3189) used 2152 FORTA PIM (mean $0.9 \pm 1.03$ per patient), 936 PRISCUS PIM $(0.3 \pm 0.58)$ and 4311 EU(7)-PIM (1.4 \pm 1.29$)$. The most common FORTA PIM was phenprocoumon (13.8\%); the most prevalent PRISCUS PIM was amitriptyline (2.8\%); the most common EU(7)-PIM was omeprazole (14.0\%). The lists rate PIM differently, with an overall overlap of $6.6 \%$. Increasing use of PIM is significantly associated with reduced cognitive function that was detected with a correlation coefficient of -0.60 for FORTA PIM ( $p=0.002),-0.72$ for PRISCUS PIM $(p=0.025)$ and -0.44 for EU(7)-PIM ( $p=0.005)$.

Conclusion We identified PIM using FORTA, PRISCUS and EU(7)-PIM lists differently and found that PIM use is associated with cognitive impairment according to LDST, whereby the FORTA list best explained cognitive decline for the German population. These findings are consistent

\section{STRENGTH AND LIMITATIONS OF THIS STUDY}

$\Rightarrow$ From 3189 multimorbid elderly patients, medication was recorded using brown bag review, taking into account not only prescription medicines but also over-the-counter medicines.

$\Rightarrow$ Drugs were categorised independently of dose according to PRISCUS and EU(7)-potentially inappropriate medication (PIM) lists because the daily dose and the frequency were not sufficiently documented.

$\Rightarrow$ Since the FORTA list does not differentiate between drugs on demand or drugs taken regularly, all drugs were included in the analysis to allow comparability between the three PIM lists.

$\Rightarrow$ The multivariate analysis and the multilevel models allow cluster effects.

with a negative impact of PIM use on multimorbid elderly patient outcomes.

Trial registration number ISRCTN89818205.

\section{INTRODUCTION}

Medication management in multimorbid elderly patients is becoming more and more relevant because of ageing populations worldwide. Due to alterations in pharmacokinetics and pharmacodynamics, the risk for adverse drug events (ADEs) in older adults is increased. ${ }^{1}{ }^{2}$ In addition, elderly patients often suffer from multiple chronic conditions leading to a higher risk of hospitalisation. ${ }^{34}$ Another risk factor in elderly patients is multimedication that is associated with 
multimorbidity. ${ }^{5}$ Multimedication, also known as "polypharmacy', is defined as the coprescription of at least five drugs ${ }^{78}$ and leads to a higher risk of drug-druginteractions, drug-disease interactions and medication errors. ${ }^{9-11}$ Moreover, elderly patients are especially vulnerable to potentially inappropriate medication (PIM), which is associated with decreased cognitive skills, frailty and falls. ${ }^{12}$ The identification of PIM is an important step to improve medication safety and to optimise prescribing and also deprescribing in multimorbid elderly patients. Since the first PIM list was published by Beers, several tools from different countries have been published. ${ }^{13}$ In our study, we decided to compare the following three screening tools, all of which are well suited for the German pharmaceutical market: FORTA (Fit fOR The Aged), PRISCUS (Latin for 'time-honoured') and EU(7)-PIM lists. ${ }^{14-16}$ We wanted to compare a well-established, but 10-year-old, German national PIM list (PRISCUS) with a more currently updated and more comprehensive European PIM list (EU(7)-PIM list). As both lists are explicit tools and do not focus on individual patient's needs, we decided to include FORTA list as an implicit national PIM list in our analysis. The PIM lists provide a broad heterogeneity of the PIM included, which poses the question of whether they rate drugs similarly.

Although studies are examining PIM use in Germany using PRISCUS and EU(7)-PIM list, only a few offer large data from community-dwelling patients, and they do not investigate the association of PIM use on the cognitive function. ${ }^{17-19}$ In addition, large studies often only use health insurance data and do not include over-the-counter (OTC) medication. ${ }^{20}$ To the best of our knowledge, no study compares FORTA as an implicit PIM list with the two explicit PIM lists PRISCUS and EU(7)-PIM list for the same patient collective, especially concerning the association with a decrease in cognitive function. Since the risk of developing ADEs is even higher in patients with cognitive impairments. ${ }^{21}$

The prospective German cohort study MultiCare was conducted to investigate the consequences of multimorbidity in elderly patients in primary care. As part of MultiCare, general practitioners (GPs) and patients were interviewed about prescribed and OTC medications as well as health and functional status, thus enabling the present study. ${ }^{22}$ The aims of the current study were (1) to examine and compare the frequency of PIM use identified via three different lists-FORTA list, PRISCUS list and EU(7)-PIM list; and (2) to examine and compare associations between PIM use according to each PIM list and to identify the PIM list that has the most impact on cognitive function.

\section{METHODS}

\section{Study design}

MultiCare-a multicentre, observational longitudinal cohort-recruited multimorbid patients from a total of 158 general practices in eight study centres in Germany
(Universities of Bonn, Düsseldorf, Frankfurt/Main, Hamburg, Jena, Leipzig, Mannheim and Munich). Patients were randomly selected from the electronic files of GPs. Patient inclusion criteria were:at least three diagnosed chronic diseases and age between 65 and 85 years. Patients were excluded if they were nursing home residents; were blind and deaf; could not provide consent, particularly patients with dementia; had an expected life expectancy of fewer than 3 months; had insufficient ability to read and speak German; participated in other studies; and were poorly known by the physician. Out of 50786 patients from the GP's electronic files, a random sample of 7172 were contacted for informed consent after screening for inclusion and exclusion criteria because of an estimated positive response rate of $40 \%-50 \%$. Of those contacted, a total of 3317 (46.2\%) responded and were willing to participate. A total of 128 patients were excluded retrospectively because they died before the baseline interview or due to other reasons. A total of 3189 patients were therefore included in our analyses. Morbidity was assessed in standardised GP interviews. Gender, age, education and income were collected in standardised patient interviews, which also comprised cognitive testing, using the letter digit substitution test (LDST). A brown bag review-gaining information about product name, German national drug code, pharmaceutical form, partly dosage, frequency and medication on demand or daily use-was performed at the patients' home, collecting information about OTC and prescription drugs used by the patients within the last 3 months. The analyses presented here are based on the baseline assessment, which took place between 21 July 2008 and 6 November 2009. Detailed information on the study design has been published previously by Schäfer $e t a t^{22} 23$ in the study protocol (online supplemental material 1).

\section{FORTA list}

The FORTA list, recently updated in 2018, includes 296 drugs used in the treatment of 30 diagnoses or indications. For each indication, the drugs were categorised by an expert Delphi panel into four categories: A (absolute), B (beneficial), C (careful) and D (don't). FORTA categories $\mathrm{A}$ and $\mathrm{B}$ are designed to detect potential undertreatment, whereas categories $\mathrm{C}$ and D signify drugs with questionable safety and effectiveness and can be used to identify PIM. $^{14}$

\section{PRISCUS list}

The German PRISCUS list (last updated in 2011) includes 83 drugs from 18 drug classes. The list provides advice on treatment alternatives and necessary actions if the PIM use is unavoidable. The PRISCUS list was developed following a structured expert survey in a two-round Delphi process. ${ }^{15}$

\section{EU(7)-PIM list}

The EU(7)-PIM list is a European list for PIM based on different national PIM lists (German PRISCUS list, PIM 
lists from USA, France and Canada) published in 2015. The EU(7)-PIM list contains 282 drugs from 34 drug classes. The list comprises restrictions to dose and duration for some drugs and gives therapeutic alternatives and advice on dose adjustment. Two Delphi survey rounds with 30 experts were performed. ${ }^{16}$

\section{PIM classification}

Data on prescription and OTC drugs were gathered via brown bag review. The drugs were classified according to the anatomical therapeutic classification (ATC) system. ${ }^{24}$

Excel V.2016 and QlikView V.11.20 (QlikTech, Radnor, USA) were used to identify PIM according to the three different PIM lists.

Drugs were classified as potentially inappropriate according to PRISCUS and EU(7)-PIM list doseindependently. As the FORTA list does not differentiate between medication on demand or daily use, we decided to include all drugs into the analysis to enable comparability between the three lists. With FORTA, we screened patients' medication for FORTA A-D drugs. In contrast to the other two lists, FORTA classification depends on the diagnosis, so that several drugs are classified differently according to their indication. When there was no documented indication for the drug and the drug only occurred once in the FORTA list, we assumed the drug was taken for that indication. Where a drug has multiple entries, we only rated the drug as PIM (C and D), if an indication was documented.

\section{Descriptive analysis of PIM use and subgroup analyses}

Data analysis was conducted using Excel V.2016 and Stata V.15.1. Subgroups were selected according to gender, age ( $<80$ years and $\geq 80$ years) and the number of drugs used (median split: 0-7 drugs and 8-29 drugs). For each subgroup, we considered the number of PIMs used per person. To examine differences in PIM use by gender, age and the number of drugs used, a two-sample t-test with equal variances was performed.

\section{Association of PIM use with cognitive function}

A multivariate mixed-effect linear regression was performed to examine the associations between each of the three different PIM lists with cognitive function. The cognitive skills were determined via LDST. ${ }^{25}$ The LDST is a speed-dependent cognitive task where patients have to replace letters by numbers in a specified time, as processing speed is an important cognitive ability for normal cognitive development and aging. ${ }^{26}$ To account for regional variation between the eight different study centres (Bonn, Düsseldorf, Frankfurt/Main, Hamburg, Jena, Leipzig, Mannheim and Munich) and personal prescribing habits of the 158 general practices, we conducted a multilevel linear regression analysis adjusted for random effects on the study centre and GP practice with study centre level. We included gender, age, number of drugs used, number of diseases weighted by severity, highest education degree in the three groups according
Table 1 Sociodemographic data of 3189 patients at baseline

\begin{tabular}{|c|c|}
\hline Age (years) (mean $\pm S D)$ & $74.4 \pm 5.2$ \\
\hline Male (years) (mean $\pm S D)$ & $74.0 \pm 5.1$ \\
\hline Female (years) (mean \pm SD) & $74.7 \pm 5.3$ \\
\hline \multicolumn{2}{|l|}{ Gender (\%) } \\
\hline Male & 40.7 \\
\hline Female & 59.3 \\
\hline \multicolumn{2}{|l|}{ Education (in CASMIN grade) (\%) } \\
\hline Low (grade 1) & 62.3 \\
\hline Medium (grade 2) & 26.8 \\
\hline High (grade 3) & 10.9 \\
\hline $\begin{array}{l}\text { Household-size adjusted net income } \\
\text { per month }(€)(\text { mean } \pm S D)\end{array}$ & $1412 \pm 704$ \\
\hline $\begin{array}{l}\text { Number of chronic conditions } \\
\text { (mean } \pm \text { SD) }\end{array}$ & $7.0 \pm 2.5$ \\
\hline Number of taken drugs (mean $\pm S D$ ) & $7.7 \pm 3.9$ \\
\hline
\end{tabular}

to the international CASMIN (Comparative Analysis of Social Mobility in Industrial Nations) classification and household net adjusted disposable income as independent variables into the model. ${ }^{27}$ The missing values in LDST, number of diseases weighted by severity, education standard and the income data sets were imputed via hot-deck imputation. This procedure has been described in detail elsewhere. ${ }^{22}$ Analyses were performed with the imputed data sets. To determine which PIM list has the most impact on the cognitive decline, the described model was extended to all three PIM lists. An alpha level of $5 \%(\mathrm{p} \leq 0.05)$ was defined as statistically significant. All statistical tests were conducted using Stata V.15.1.

\section{Patient and public involvement statement}

No patients were involved in setting the research question or the outcome measures, nor were they involved in developing plans for the design or implementation of the study. No patients were asked to advise on interpretation or writing up of results. There are no plans to disseminate the results of the research to study participants or the relevant patient community.

\section{RESULTS}

\section{Characterisation of the study cohort}

Table 1 describes the sociodemographic data of the patients. In the MultiCare cohort (3198 patients aged 65-85 years), 24535 drugs, thereof $24.2 \%$ (5935) OTC, were identified and related to an ATC code (mean 7.7 \pm 3.9 drugs; median 7 drugs, range 0-29 drugs).

As shown in table 2, patients used PIM according to FORTA with a prevalence of $55.9 \%$. In average, they used $0.9( \pm 1.0)$ FORTA PIM with a range of 0-7 PIMs per patient. PRISCUS PIMs were detected with a prevalence of $24.7 \%$, and patients used PRISCUS PIM with a mean of $0.3( \pm 0.58)$ and with a range of $0-4$. According to the 
Table 2 Comparison of the descriptive results from FORTA, PRISCUS and EU(7)-PIM list

\begin{tabular}{llll}
\hline & FORTA PIM & PRISCUS PIM & EU(7)-PIM \\
\hline Medication & & & \\
\hline Total number (\%) of drugs used & 24535 & 24535 & 24535 \\
$\quad$ Prescribed & $18600(75.8)$ & $18600(75.8)$ & $18600(75.8)$ \\
\hline OTC & $5935(24.2)$ & $5935(24.2)$ & $5935(24.2)$ \\
Total number (\%) of PIM & $2852(11.6)$ & $963(3.9)$ & $4311(17.6)$ \\
$\quad$ Prescribed & $2474(86.7)$ & $939(97.5)$ & $3919(90.9)$ \\
OTC & $378(13.3)$ & $24(2.5)$ & $392(9.1)$ \\
Median number of PIM (range) & $1(0-7)$ & $0(0-4)$ & $1(0-8)$ \\
Mean number of PIM (SD) & $0.9( \pm 1.0)$ & $0.3( \pm 0.6)$ & $1.4( \pm 1.3)$ \\
Patients (\%) & & & \\
Patients with at least one PIM & 55.9 & 24.7 & 70.1 \\
\hline Patients with one PIM & $1048(32.9)$ & $656(20.6)$ & $1020(32.0)$ \\
Patients with two PIMs & $496(15.6)$ & $123(3.9)$ & $678(21.3)$ \\
Patients with three PIMs & $168(5.3)$ & $15(0.5)$ & $320(10.0)$ \\
Patients with four PIMs & $47(1.5)$ & $4(0.1)$ & $140(4.4)$ \\
\hline Patients with five PIMs & $19(0.6)$ & - & $50(1.6)$ \\
\hline Patients with six PIMs & $3(0.1)$ & - & $19(0.6)$ \\
\hline Patients with seven PIMs & $1(0.03)$ & - & $5(0.2)$ \\
\hline Patients with eight PIMs & - & - & $2(0.1)$
\end{tabular}

FORTA, Fit fOR The Aged; OTC, over-the-counter; PIM, potentially inappropriate medication.

EU(7)-PIM list, patients used $1.4( \pm 1.29)$ with a range of 0-8 PIMs. We detected EU(7)-PIM with a prevalence of $70.1 \%$.

Regarding FORTA, we detected 2852 category C or D PIM in total and thereof $13.3 \%$ (378) OTC drugs. Divided by category, we identified a mean of 0.7 in category C FORTA PIM and 0.2 in category D FORTA PIM per patient. The most common category C PIM is phenprocoumone for the treatment of atrial fibrillation (441 patients, $13.8 \%$ ), followed by ginkgo leaf preparations for the treatment of dementia $(152,4.8 \%)$, glimepiride for the treatment of diabetes mellitus $(144,4.5 \%)$ and verapamil for the treatments of hypertension and atrial fibrillation $(116,3.6 \%)$. The most common category D drugs are acetylic salicylic acid as antiplatelet agent for atrial fibrillation with $3.1 \%$ (100 patients), molsidomine $(76,2.4 \%)$, glibenclamide $(69,2.2 \%)$ and tocopherol $(63,2.0 \%)$.

We detected 963 PRISCUS PIM in total with a proportion of $2.5 \%$ (24) OTC drugs. The most common drugs were amitriptyline (88 patients, $2.8 \%$ ), acetyldigoxin (60 patients, $1.9 \%$ ), nifedipine (53 patients, $1.7 \%$ ) and zopiclone (47 patients, $1.5 \%$ ).

We identified 4311 drugs (17.6\%) as EU(7)-PIM, and thereof $9.1 \%$ (392) were classified as OTC drugs. The most common drugs are omeprazole (448 patients, $14.0 \%)$, diclofenac (390 patients, 12.2\%), ibuprofen (335 patients, $10.5 \%$ ) and acetyl salicylic acid as analgesic (191, 6.0\%).

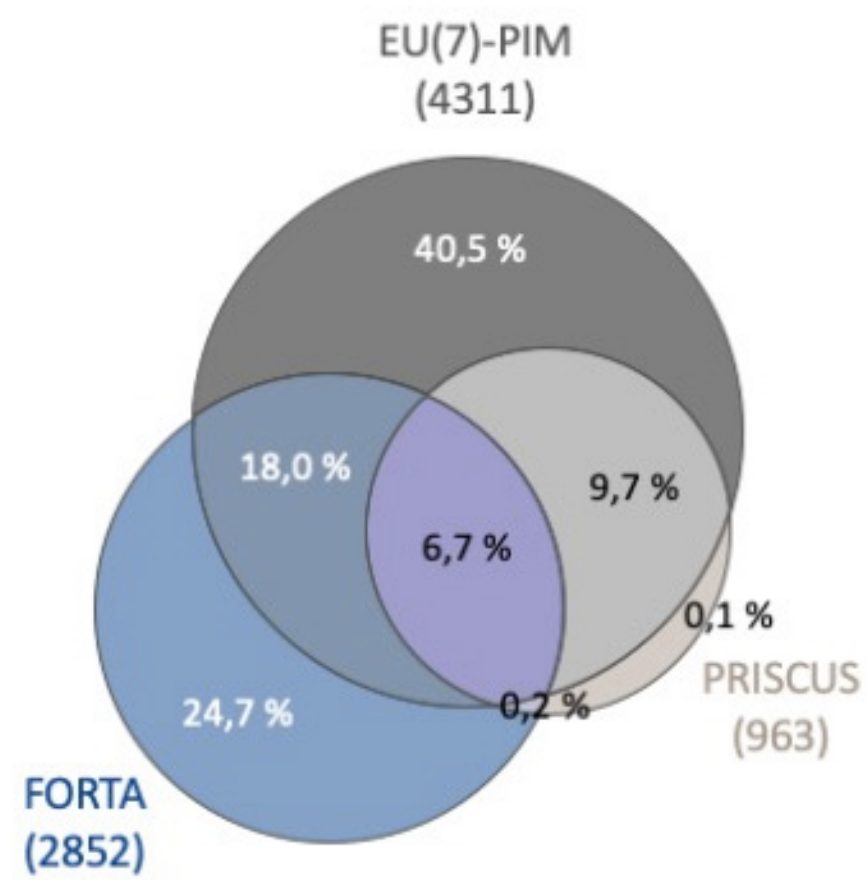

Figure 1 Venn diagram showing the overlap between FORTA, PRISCUS and EU(7)-PIM lists in terms of PIM (percentages sum up to 100\%). FORTA, Fit fOR The Aged; PIM, potentially inappropriate medication. 
Table 3 Top 20 drugs most commonly resulting in inappropriate prescribing according to FORTA, PRISCUS and EU(7)-PIM (drugs analysed in total 24 535)

\begin{tabular}{llll}
\hline PIM & FORTA & PRISCUS & Eu(7)-PIM \\
\hline Omeprazole & - & - & 448 \\
\hline Phenprocoumon & 441 & - & - \\
\hline Diclofenac & - & - & 390 \\
\hline Ibuprofen & - & - & 335 \\
\hline Acetyl salicylic acid & - & - & 191 \\
(analgesic) & & & \\
\hline Glimepiride & 144 & - & 144 \\
\hline Pantoprazole & - & - & 157 \\
\hline Ginkgo biloba & 152 & - & - \\
leaves & & - & 144 \\
\hline Glimepiride & 144 & - & 116 \\
\hline Verapamil & 116 & - & 114 \\
\hline Moxonidine & 114 & - & 107 \\
\hline Spironolactone & - & - & 105 \\
\hline Tramadol & 105 & - & 104 \\
\hline Theophylline & 104 & - & - \\
\hline Acetyl salicylic acid \\
(antiplatelet agent)
\end{tabular}

Figure 1 illustrates the overlap between the three different PIM lists. Of the detected PIMs, 384 (6.7\%) were identified within all three lists, while nearly all PRISCUS PIMs were also detected by EU(7)-PIM (97.9\%). Moreover, the summary of the top 20 PIMs used by the patients points out the small grade of overlap between the three PIM lists (table 3).

\section{Subgroup analysis: age, gender and number of used drugs}

Results of the subgroup analysis are described in table 4 . Women were using significantly more PIM according to PRISCUS and EU(7)-PIM list (both $\mathrm{p}<0.001$ ). Furthermore, patients who are 80 years old and older used more PIM according to FORTA and PRISCUS list ( $\mathrm{p}=0.005$ and $\mathrm{p}<0.001)$. In addition, we detected that patients using more than seven drugs at the same time used significantly more PIM according to all three PIM lists (all lists $\mathrm{p}<0.001)$. This effect was also detectable-in all three PIM lists-continuously with a growing number of used drugs (FORTA: 0.130, 95\% CI 0.122 to $0.138, \mathrm{p}<0.001$; PRISCUS: $0.050,95 \%$ CI 0.045 to 0.055 , $\mathrm{p}<0.001$; $\mathrm{EU}(7)$ : $0.190,95 \%$ CI 0.181 to $0.200, \mathrm{p}<0.001)$.

\section{Association with cognitive function}

In table 5, the results of the LDST are shown. On average, patients achieved a mean score of $23( \pm 7.1)$ with a range of $0-50$ in LDST. More than half of the patients (51.9\%) scored between 20 and 29 in LDST.

We found that patients who used PIM scored significantly worse values in LDST than patients who used less or none PIM. This is true for all three PIM lists in a multivariate approach, with each PIM list analysed separately in the same regression model: FORTA-PIM $(-0.397,95 \%$ CI -0.644 to $-0.150, p=0.002)$, PRISCUS $(-0.464,95 \%$ CI -0.870 to $-0.058, \mathrm{p}=0.025)$ and $\mathrm{EU}(7)-\mathrm{PIM}(-0.300,95$ CI\% -0.508 to $-0.092, \mathrm{p}=0.005$ ) (table 6).

By including all three PIMs in one regression model, the impact of FORTA $(-0.306,95 \%$ CI -0.567 to -0.044 , $\mathrm{p}=0.022)$, PRISCUS $(-0.118,95 \%$ CI -0.652 to 0.276 , $\mathrm{p}=0.428)$ and $\mathrm{EU}(7)-\mathrm{PIM}$ list $(-0.188,95 \%$ CI -0.416 to $0.072, p=0.168$ ) on the cognitive decline is shown (table 7). It appears that the association between PIM use and the patient's ability to complete the LDST is best depicted in the FORTA list.

\section{DISCUSSION}

\section{Statement of principal findings}

With the help of the three PIM lists, different numbers of PIM within the multimorbid elderly patients were detected. We identified that the use of PIM is associated with reduced cognitive function in multimorbid elderly patients. This was demonstrated for FORTA, PRISCUS and EU(7)-PIM lists. However, the FORTA list seems to be most suitable to reveal the association between PIM use and decreased cognitive function in multimorbid elderly patients.

\section{PIM classification}

In general, our data are in good accordance with recently published data. Other studies detected EU(7)-PIM with a prevalence of $57.2 \%-72.8 \% .{ }^{128} 29$ This seems to be comparable with our findings $(70.1 \%)$. None of the three studies included OTC drugs, and in addition, Wauters et $a l$ included only patients 80 years and older.

The German ESTHER cohort detected PIM according to PRISCUS with a prevalence of $13.7 \%$ and according to the EU(7)-PIM list of $37.4 \% .{ }^{19}$ As they have a much younger patient collective- 50 years- 75 years-it is explainable that we found a higher prevalence in the MultiCare cohort (24.7\% prevalence of PRISCUS PIM). Another reason for the higher observed PIM prevalences in detected EU(7)-PIM and PRISCUS PIM might be that only patients with at least three chronic diseases were included in our study. This is also apparent in a study including only patients with multimedication (five or more drugs) where the authors detected even higher prevalences of $45 \%$ PRISCUS PIM and $61 \%$ FORTA PIM (prevalence of FORTA PIM in our study: 55.9\%). ${ }^{30}$ The observed prevalence for PRISCUS PIM is quite lower than for FORTA and EU(7)-PIM. This is also following 
Table 4 Difference between gender (male and female), the two different age groups ( $<80$ years and $\geq 80$ years) and between patients with zero to seven drugs at the same time and patients using more than seven drugs (8-29) at the same time measured with FORTA, PRISCUS and EU(7)-PIM list (significant $p$ values marked in bold)

\begin{tabular}{|c|c|c|c|c|c|c|c|c|}
\hline & & \multirow[b]{2}{*}{ Patients (n) } & \multicolumn{6}{|l|}{ PIM absolute } \\
\hline & & & (PIM (n)/drugs (n) (\%)) & Mean per patient & SD & Range & $95 \% \mathrm{Cl}$ & $P$ value \\
\hline \multirow[t]{2}{*}{ FORTA PIM } & Male & 1271 & $1126(4.6)$ & 0.89 & 1.00 & $0-5$ & 0.83 to 0.94 & \\
\hline & & & & & & & & 0.362 \\
\hline PRISCUS PIM & Male & 1271 & $336(1.4)$ & 0.26 & 0.55 & $0-4$ & 0.23 to 0.29 & \\
\hline \multirow[t]{3}{*}{ EU (7)-PIM } & Male & 1271 & $1538(6.3)$ & 1.21 & 1.22 & $0-7$ & 1.14 to 1.28 & \\
\hline & Female & 1876 & $2773(11.3)$ & 1.48 & 1.33 & $0-8$ & 1.42 to 1.54 & \\
\hline & & & & & & & & $<0.001$ \\
\hline FORTA PIM & $<80$ years & 2601 & $2293(9.3)$ & 0.88 & 1.02 & $0-7$ & 0.84 to 0.92 & \\
\hline \multirow{2}{*}{ PRISCUS PIM } & $\geq 80$ years & 546 & $219(0.9)$ & 0.40 & 0.62 & $0-3$ & 0.34 to 0.45 & \\
\hline & & & & & & & & $<0.001$ \\
\hline \multirow[t]{3}{*}{ EU (7)-PIM } & $<80$ years & 2601 & $3519(14.3)$ & 1.35 & 1.29 & $0-8$ & 1.30 to 1.40 & \\
\hline & $\geq 80$ years & 546 & $792(3.2)$ & 1.44 & 1.31 & $0-8$ & 1.33 to 1.55 & \\
\hline & & & & & & & & 0.145 \\
\hline \multirow[t]{3}{*}{ FORTA PIM } & $0-7$ drugs & 1646 & $857(3.5)$ & 0.52 & 0.70 & $0-3$ & 0.49 to 0.55 & \\
\hline & 8-29 drugs & 1501 & 1995 (8.1) & 1.32 & 1.16 & $0-7$ & 1.27 to 1.39 & \\
\hline & & & & & & & & $<0.001$ \\
\hline PRISCUS PIM & $0-7$ drugs & 1646 & $271(1.1)$ & 0.16 & 0.41 & $0-2$ & 0.14 to 0.18 & \\
\hline
\end{tabular}

FORTA, Fit fOR The Aged; PIM, potentially inappropriate medication.

Table 5 Descriptive results of LDST (imputed data: missing value 255 from 3189 patients) to measure the cognitive function of patients and the number of boxes patients were able to fill out correctly

\begin{tabular}{|ll|}
\hline & LDST \\
\hline Mean (SD) & $23.0( \pm 7.1)$ \\
\hline Median (range) & $23(0-50)$ \\
\hline Results LDST & Patients (n) \\
\hline$\leq 10$ (relative) & $78(2.4 \%)$ \\
\hline 10 up to $<20$ (relative) & $879(27.6 \%)$ \\
\hline 20 up to $<30$ (relative) & $1656(51.9 \%)$ \\
\hline 30 up to $<40$ (relative) & $533(16.7 \%)$ \\
\hline$\geq 40$ (relative) & $43(1.3 \%)$ \\
\hline
\end{tabular}

LDST, letter digit substitution test. the aforementioned literature. These differences in the detected prevalences might occur due to the smaller number of drugs included in the PRISCUS list.

Moreover, the three PIM lists evaluate the drugs quite differently. For example, the PRISCUS list comprises more 'classic inappropriate' substances like antidepressants $(18.2 \%)$, antihypertensives $(5.7 \%)$ and hypnotics/ sedatives $(13.5 \%)$, which is also in good accordance with findings described in literature. ${ }^{317}$ It is described that the prescriptions of sedatives, hypnotics, neuroleptics, antipsychotics and antihypertensives are risk factors for falls in elderly patients. ${ }^{32}$ On the contrary, in addition to the classic potentially inappropriate substances, the FORTA list also identified as PIM those whose effectiveness had not been proven, for example, Ginkgo biloba and tocopherol. In addition, phenprocoumon is only listed in FORTA but not in PRISCUS or EU-(7) PIM, and it was the most common FORTA PIM. In V.2015 of FORTA, 
Table 6 Multivariate linear regression model-impact of FORTA or PRISCUS or EU(7)-PIM use on cognitive function measured by LDST

\begin{tabular}{|c|c|c|c|}
\hline LDST & Correlation coefficient & $P$ value & $95 \% \mathrm{Cl}$ \\
\hline FORTA PIM per patient & -0.397 & 0.002 & -0.644 to -0.150 \\
\hline Age & -0.340 & $<0.001$ & -0.383 to -0.296 \\
\hline Sex & 2.538 & $<0.001$ & 2.072 to 3.004 \\
\hline CASMIN3_2 & 2.348 & $<0.001$ & 1.813 to 2.883 \\
\hline CASMIN3_3 & 3.791 & $<0.001$ & 3.007 to 4.575 \\
\hline Income & 2.407 & $<0.001$ & 1.869 to 2.945 \\
\hline Number of diseases weighted by severity & -0.121 & $<0.001$ & -0.169 to -0.072 \\
\hline Number of taken drugs & -0.034 & 0.340 & -0.105 to 0.036 \\
\hline PRISCUS PIM per patient & -0.464 & 0.025 & -0.870 to -0.058 \\
\hline Age & -0.340 & $<0.001$ & -0.383 to -0.296 \\
\hline Sex & 2.560 & $<0.001$ & 2.093 to 3.026 \\
\hline CASMIN3_2 & 2.366 & $<0.001$ & 1.831 to 2.901 \\
\hline CASMIN3_3 & 3.776 & $<0.001$ & 2.992 to 4.561 \\
\hline Income & 2.423 & $<0.001$ & 1.885 to 2.961 \\
\hline Number of diseases weighted by severity & -0.127 & $<0.001$ & -0.176 to -0.079 \\
\hline Number of taken drugs & -0.060 & 0.079 & -0.127 to 0.007 \\
\hline EU(7)-PIM per patient & -0.300 & 0.005 & -0.508 to -0.092 \\
\hline Age & -0.344 & $<0.001$ & -0.387 to -0.300 \\
\hline Sex & 2.597 & $<0.001$ & 2.130 to 3.065 \\
\hline CASMIN3_2 & 2.351 & $<0.001$ & 1.815 to 2.886 \\
\hline CASMIN3_3 & 3.772 & $<0.001$ & 2.998 to 4.556 \\
\hline Income & 2.409 & $<0.001$ & 1.871 to 2.947 \\
\hline Number of diseases weighted by severity & -0.127 & $<0.001$ & -0.176 to -0.079 \\
\hline Number of taken drugs & -0.025 & 0.507 & -0.101 to 0.050 \\
\hline
\end{tabular}

Dependent variable: results from LDST; independent variables: FORTA PIM or PRISCUS PIM or EU(7)-PIM; covariables included in the regression model: sex, age, education standard (casmin3_2: comparison between medium and low educational standard; casmin3_3: comparison between high and low educational standard), number of diseases weighted by severity, income and number of taken drugs. Every PIM list is analysed separately in the same regression model.

FORTA, Fit fOR The Aged; LDST, letter digit substitution test; PIM, potentially inappropriate medication.

phenprocoumon was listed as a class B drug. However, with the availability of newer anticoagulants that do not require INR (international normalized ratio) monitoring, it has been recategorised as a FORTA C PIM in the updated version from 2018. Nevertheless, in most guidelines, phenprocoumon is still recommended. ${ }^{33}$

In addition to the substances detected with the PRISCUS list mentioned previously, proton pump inhibitors (PPIs) $(15.7 \%)$ and NSAIDs (non-steroidal anti-inflammatory drug) $(18.7 \%)$ were found to be the most common PIM in the EU(7)-PIM list. The high use of PPIs and NSAIDs is shown by some other studies as well. ${ }^{128}$ But interestingly, in most studies, hypnotics and sedatives were found to be the most commonly prescribed EU(7)-PIM. We detected only $3.6 \%$ benzodiazepines, whereas other studies detected $4.2 \%-18.1 \%$ benzodiazepines, and therefore some studies presented only single substances. ${ }^{1}{ }^{28}{ }^{34}$ Strikingly, there was only a small overlap between all three PIM lists. Although all three lists were developed for the German or European drug market, there is-besides some classic drugs-a broad heterogeneity in detected PIM. That raises the question of whether the use of only one PIM list is sufficient for the identification of PIM, leading to the assumption that already existing PIM lists need to be improved or even questioned to simplify and standardise this process. We need valid tools for identifying PIM because medication management in elderly multimorbid patients is highly complex. Detecting PIM and showing alternatives are still an important step to improve medication safety in multimorbid elderly patients. ${ }^{12}$

\section{Risk factors for PIM use}

To minimise the amount of prescribed PIM, we need to find out and, if possible, reduce risk factors for prescribing PIM. For example, we could demonstrate that multimedication is a risk factor for prescribing PIM in multimorbid elderly patients by pointing out that patients using seven drugs and more at the same time used significantly more 
Table 7 Multivariate linear regression model-impact of FORTA, PRISCUS and EU(7)-PIM use on cognitive skills measured by LDST

\begin{tabular}{|c|c|c|c|}
\hline LDST & Correlation coefficient & $P$ value & $95 \% \mathrm{Cl}$ \\
\hline FORTA PIM per patient & -0.306 & 0.022 & -0.567 to -0.044 \\
\hline PRISCUS PIM per patient & -0.118 & 0.428 & -0.652 to 0.276 \\
\hline Age & -0.172 & $<0.001$ & -0.384 to -0.296 \\
\hline Sex & -0.340 & $<0.001$ & 2.114 to 3.050 \\
\hline Income & 2.406 & $<0.001$ & 1.868 to 2.943 \\
\hline Number of diseases weighted by severity & -0.121 & $<0.001$ & -0.170 to -0.073 \\
\hline Number of taken drugs & -0.004 & 0.921 & -0.081 to 0.073 \\
\hline \multicolumn{4}{|c|}{$\begin{array}{l}\text { Dependent variable: results from LDST; independent variables: FORTA PIM, PRISCUS PIM, EU(7)-PIM; covariables included in the regression model: } \\
\text { sex, age, education standard (casmin3_2: comparison between medium and low educational standard, casmin3_3: comparison between high and } \\
\text { low educational standard), number of diseases weighted by severity, income and number of taken drugs. } \\
\text { All three PIM lists are included in one regression model. } \\
\text { FORTA, Fit fOR The Aged; LDST, letter digit substitution test; PIM, potentially inappropriate medication. }\end{array}$} \\
\hline
\end{tabular}

PIM. As multimedication is a well-described risk factor for prescribing PIM and is also associated with a higher risk of falls and hip fractures in multimorbid elderly patients, we must have the goal of rational prescribing. ${ }^{31} 3536$ Furthermore, the association between age and PIM use is inconsistent between the three PIM lists. Previous publications also showed different results. ${ }^{21}$ We were also able to demonstrate that women are at higher risk of receiving PIM according to PRISCUS and EU(7)-PIM list but not according to FORTA. The observed gender differences in PIM use are in good accordance with the literature. ${ }^{19} 31$ Moreover, Toepfer et al indicate that the female sex is at a greater risk of PIM use due to higher use of antidepressants, sleep-inducing drugs, analgesics and the use of oestrogens. $^{17}$

\section{Association of the use of PIM with the cognitive function}

The relation between PIM use and the reduction of the cognitive function was based on poorer scores in a LDST, as determined by multivariate analysis. Other studies described that age, educational standard and gender influence LDST test results, so we decided-among othersto include those variables in our regression model. ${ }^{25} 37$ In addition, the prescribing bias due to regional effects and the GPs were minimised by performing a multilevel regression. Muhlack et $a l^{19}$ showed a strong cognitive impairment that is associated with PIM use according to EU (7), PRISCUS and Beers list. Beyond that, most studies that revealed the influence of PIM on cognitive function included patients with dementia. ${ }^{21} 38$ Patients with dementia already have cognitive impairments due to their illness. In the MultiCare study, we excluded patients with all forms of dementia. Even though we cannot completely exclude the presence of already cognitively impaired patients in our study, we can show an effect of PIM use on the cognitive decline with less interference due to already cognitively impaired patients. Interestingly, in our model, the FORTA list best explained the decrease in cognitive function in multimorbid elderly patients. A possible explanation is that the FORTA list-in contrast to the PRISCUS and EU(7)-PIM lists-rates drugs based on indications as an implicit PIM list. Most other PIM lists are explicit tools and do not address the individual differences in patient needs. Furthermore, the FORTA list was developed for the German drug market and in contrast to the also German PRISCUS list, the FORTA list was recently updated in 2018. Another advantage is that the FORTA list is basically a positive and negative list because of the different categories. Also, the VALFORTA study points out the benefits of the FORTA list by showing that the use of the FORTA list reduced the occurrence of $\mathrm{ADE}$ and revealed overtreatment and undertreatment in elderly patients. ${ }^{39}$

\section{Strength and limitations}

There are some limitations with the present study. The PIM use according to the PRISCUS and EU(7)-PIM lists might have been overestimated in our study because we did not differentiate between medication on demand and regularly used medication. For example, omeprazole is inappropriate if it is used at maximum dosage for longer than 8 weeks without a clear indication. Furthermore, the daily dose was not documented consequently during the brown bag review. Due to this fact, it was not possible to categorise PIM-for the PRISCUS and EU(7)-PIM listsaccording to their dosing.

The medication review was conducted at the patients' homes, via brown bag review. Additionally, patients were asked how the GPs told them to take their medication. It is therefore possible that a daily used NSAID, hypnotic, or PPIs has been labelled as medication on demand. As we did not want to underestimate the use of critical drugs like PPIs, NSAIDs or hypnotics, we decided to count every drug that is documented as a PIM according to one of 
the three lists. In addition, almost all studies using large data analysed PIM irrespective of the dose, that is why we believe that this procedure is suitable for our patient collective. ${ }^{17-20}$ However, this may lead to an overestimation of PIM use according to the PRISCUS and EU(7)-PIM lists. Therefore, we conducted a sensitivity analysis, excluding all medication on demand for PRISCUS and EU(7)-PIM. Both analyses still showed that there is an association between PIM according to the PRISCUS $(-0.497,95 \%$ CI -0.942 to $-0.051, \mathrm{p}=0.029)$ and $\mathrm{EU}(7)-\mathrm{PIM}(-0.391,95 \%$ CI -0.610 to $-0.172, \mathrm{p}<0.001)$ lists and a decreased cognitive function. Including all three PIM lists without medication on demand in one model, besides FORTA also the EU (7)-PIM list shows a significant association on patients' cognitive functions (FORTA: CC $-0.269,95 \%$ CI -0.534 to $-0.003, \mathrm{p}=0.047 ; \mathrm{EU}(7)$-PIM: $-0.275,95 \% \mathrm{CI}-0.536$ to $-0.014, \mathrm{p}=0.039)$, while PRISCUS does not $(-0.124,95 \%$ CI -0.631 to $0.382, \mathrm{p}=0.631$ ).

FORTA PIM was analysed strictly indication-based, so it is possible that we even underestimated the sensitivity of the FORTA list. A strength of the data presented is that we included OTC drugs. Among the detected FORTA PIM and EU(7)-PIM, we had a high proportion of OTC drugs $(13.3 \%$ and $9.1 \%)$. In addition, the number of OTC drugs might even have been underestimated because we counted NSAIDs and PPIs as prescription drugs because as they are prescribable for some indications due to the German medicines law. An additional strength consists of multivariate analyses, multilevel models allowing for cluster effects and advanced treatment of missing values.

Taken together, we were able to show that decreased cognitive function was apparent within all three PIM lists and that the FORTA list illustrates the cognitive decline most clearly. Besides that, the association between decreased cognitive function and the use of PIM underlines the importance of reducing the amount of PIM in elderly patients.

\section{CONCLUSION}

The supply of multimorbid elderly patients is a huge challenge we are facing, and therefore, we need to improve the medication safety of those patients. By identifying PIM with FORTA, PRISCUS and EU(7)-PIM lists and revealing that cognitive impairment is associated with PIM use, we can highlight the negative association of PIM use on elderly patients' outcomes and emphasise the importance of reducing the amount of PIM in elderly patients. To improve drug safety, it is important to have tools to identify PIM. However, the broad heterogeneity of detected PIM with the different tools also reflects that we still need to improve the already existing PIM lists.

Although we identified a high use of PIM among elderly multimorbid patients with different PIM lists, a longitudinal analysis is needed.

In summary, we identified PIM using FORTA, PRISCUS and EU(7)-PIM lists and revealed that PIM use is related to a decreased cognitive function. For the German population, the use of PIM detected by the FORTA list best explained the cognitive decline.

Author affiliations

${ }^{1}$ Hospital Pharmacy, University Medical Center Hamburg-Eppendorf, Hamburg, Germany

${ }^{2}$ Department of Primary Medical Care, University Medical Center HamburgEppendorf, Hamburg, Germany

${ }^{3}$ Department of Psychiatry, Technical University of Munich, Munchen, Germany ${ }^{4}$ Institute of General Practice, Friedrich-Schiller-Universität Jena, Jena, Germany ${ }^{5}$ Institute of General Practice and Family Medicine, Ludwig-Maximilians-Universitat Munchen, Munchen, Germany

${ }^{6}$ Institute of General Practice, Heinrich-Heine-Universitat Dusseldorf, Dusseldorf, Germany

${ }^{7}$ Department for Health Economics and Health Services Research,

Universitatsklinikum Hamburg-Eppendorf, Hamburg, Germany

${ }^{8}$ Department of Psychiatry and Psychotherapy, University of Bonn, Bonn,

Germany

${ }^{9}$ Institute of General Practice, Goethe University Frankfurt, Frankfurt am Main, Germany

${ }^{10}$ Institute for Social Medicine, Leipzig University, Leipzig, Germany

${ }^{11}$ Department of Medical Biometry and Epidemiology, University Medical Center Hamburg-Eppendorf, Hamburg, Germany

${ }^{12}$ Medical Faculty, Central Institute of Mental Health, Mannheim, Germany

${ }^{13}$ Institute for General Practice, Hannover Medical School, Hannover, Germany

${ }^{14}$ Research Department, Albertinen-Haus Zentrum für Geriatrie und Gerontologie Medizinisch-Geriatrische Klinik, Hamburg, Germany

${ }^{15}$ Department of Stem Cell Transplantation, University Medical Center HamburgEppendorf, Hamburg, Germany

\section{Twitter Martin Scherer @degampraesident}

Acknowledgements This article is on behalf of the MultiCare Cohort Study Group, which consists of Attila Altiner, Horst Bickel, Wolfgang Blank, Monika Bullinger, Hendrik van den Bussche (principal investigator), Anne Dahlhaus, Lena Ehreke, Michael Freitag, Angela Fuchs, Jochen Gensichen, Ferdinand Gerlach, Heike Hansen, Sven Heinrich, Susanne Höfels, Olaf von dem Knesebeck, Hans-Helmut König, Norbert Krause, Hanna Leicht, Margrit Löbner, Melanie Luppa, Wolfgang Maier, Manfred Mayer, Christine Mellert, Anna Nützel, Thomas Paschke, Juliana Petersen, Jana Prokein, Steffi Riedel-Heller, Heinz-Peter Romberg, Ingmar Schäfer, Martin Scherer (principal investigator), Gerhard Schön, Susanne Steinmann, Sven Schulz, Karl Wegscheider, Klaus Weckbecker, Jochen Werle, Siegfried Weyerer and Birgitt Wiese. We are grateful to the general practitioners in Bonn, Dusseldorf, Frankfurt/Main, Hamburg, Jena, Leipzig, Mannheim and Munich who supplied clinical information on their patients.

Contributors CK, IS, HvdB, HB, TD, AF, H-HK, WM, KM, SGR-H, GS, SW, BW, WvR-K, $\mathrm{CL}$ and MS provided substantial contributions to study design and implementation. The first draft of the manuscript was written by CK, and all authors commented on previous versions of the manuscript. All authors revised and approved the final manuscript.

Funding The study was funded by the German Federal Ministry of Education and Research (grant numbers 01ET0725-31 and 01ET1006A-K). The funders had no role in study design, data collection and analysis, decision to publish or preparation of the manuscript.

Competing interests None declared.

Patient consent for publication Not required.

Ethics approval The study is conducted in compliance with the Helsinki Declaration. The study protocol was approved by the ethics committee of the Medical Association of Hamburg in February 2008 and amended in November 2008 (approval number 2881).

Provenance and peer review Not commissioned; externally peer reviewed.

Data availability statement Data are available upon reasonable request. The data that support the findings of this study are available from Prof. Hendrik van den Bussche but restrictions apply to the availability of these data, which were used under license for the current study, and so are not publicly available. Data are however available from the authors upon reasonable request and with permission of Prof. Hendrik van den Bussche. 
Supplemental material This content has been supplied by the author(s). It has not been vetted by BMJ Publishing Group Limited (BMJ) and may not have been peer-reviewed. Any opinions or recommendations discussed are solely those of the author(s) and are not endorsed by BMJ. BMJ disclaims all liability and responsibility arising from any reliance placed on the content. Where the content includes any translated material, BMJ does not warrant the accuracy and reliability of the translations (including but not limited to local regulations, clinical guidelines, terminology, drug names and drug dosages), and is not responsible for any error and/or omissions arising from translation and adaptation or otherwise.

Open access This is an open access article distributed in accordance with the Creative Commons Attribution Non Commercial (CC BY-NC 4.0) license, which permits others to distribute, remix, adapt, build upon this work non-commercially, and license their derivative works on different terms, provided the original work is properly cited, appropriate credit is given, any changes made indicated, and the use is non-commercial. See: http://creativecommons.org/licenses/by-nc/4.0/.

\section{ORCID iDs}

Caroline Krüger http://orcid.org/0000-0001-9080-1562

Ingmar Schäfer http://orcid.org/0000-0002-1038-7478

\section{REFERENCES}

1 Mucalo I, Hadžiabdić MO, Brajković A, et al. Potentially inappropriate medicines in elderly hospitalised patients according to the EU(7)-PIM list, STOPP version 2 criteria and comprehensive protocol. Eur J Clin Pharmacol 2017;73:991-9.

2 Morin L, Laroche M-L, Texier G, et al. Prevalence of potentially inappropriate medication use in older adults living in nursing homes: a systematic review. J Am Med Dir Assoc 2016;17:862.e1-862.e9.

3 Endres HG, Kaufmann-Kolle P, Steeb V, et al. Association between potentially inappropriate medication (PIM) use and risk of hospitalization in older adults: an observational study based on routine data comparing PIM use with use of Pim alternatives. PLoS One 2016;11:e0146811.

4 Harrison SL, Kouladjian O'Donnell L, Bradley CE, et al. Associations between the drug burden index, potentially inappropriate medications and quality of life in residential aged care. Drugs Aging 2018;35:83-91.

5 Strehblow C, Smeikal M, Fasching P. Polypharmacy and excessive polypharmacy in octogenarians and older acutely hospitalized patients. Wien Klin Wochenschr 2014;126:195-200.

6 Fortin M, Stewart M, Poitras M-E, et al. A systematic review of prevalence studies on multimorbidity: toward a more uniform methodology. Ann Fam Med 2012;10:142-51.

7 Maher RL, Hanlon J, Hajjar ER. Clinical consequences of polypharmacy in elderly. Expert Opin Drug Saf 2014;13:57-65.

8 Hajjar ER, Cafiero AC, Hanlon JT. Polypharmacy in elderly patients. Am J Geriatr Pharmacother 2007;5:345-51.

9 Pazan F, Burkhardt $\mathrm{H}$, Frohnhofen $\mathrm{H}$, et al. Changes in prescription patterns in older hospitalized patients: the impact of FORTA on disease-related over- and under-treatments. Eur J Clin Pharmacol 2018;74:339-47.

10 O Riordan D, Aubert CE, Walsh KA, et al. Prevalence of potentially inappropriate prescribing in a subpopulation of older European clinical trial participants: a cross-sectional study. BMJ Open 2018;8:e019003.

11 Guthrie B, Makubate B, Hernandez-Santiago V, et al. The rising tide of polypharmacy and drug-drug interactions: population database analysis 1995-2010. BMC Med 2015;13:74.

12 Motter FR, Fritzen JS, Hilmer SN, et al. Potentially inappropriate medication in the elderly: a systematic review of validated explicit criteria. Eur J Clin Pharmacol 2018;74:679-700.

13 American Geriatrics Society 2012 Beers Criteria Update Expert Panel. American geriatrics Society updated beers criteria for potentially inappropriate medication use in older adults. J Am Geriatr Soc 2012;60:616-31.

14 Pazan F, Weiss C, Wehling M, et al. The EURO-FORTA (fit fOR the aged) list: international consensus validation of a clinical tool fOR improved drug treatment in older people. Drugs Aging 2018;35:61-71.

15 Holt S, Schmiedl S, Thürmann PA. Potentially inappropriate medications in the elderly: the PRISCUS list. Deutsches Ärzteblatt International 2010;107:543-51.

16 Renom-Guiteras A, Meyer G, Thürmann PA. The EU(7)-PIM list: a list of potentially inappropriate medications for older people consented by experts from seven European countries. Eur J Clin Pharmacol 2015;71:861-75.

17 Toepfer S, Bolbrinker J, König M, et al. Potentially inappropriate medication in older participants of the Berlin Aging Study II (BASE-II) - Sex differences and associations with morbidity and medication use. PLoS One 2019;14:e0226511.

18 Mielke N, Huscher D, Douros A, et al. Self-Reported medication in community-dwelling older adults in Germany: results from the Berlin initiative study. BMC Geriatr 2020;20:22.

19 Muhlack DC, Hoppe LK, Stock C, et al. The associations of geriatric syndromes and other patient characteristics with the current and future use of potentially inappropriate medications in a large cohort study. Eur J Clin Pharmacol 2018;74:1633-44.

20 Schubert I, Küpper-Nybelen J, Ihle P, et al. Prescribing potentially inappropriate medication (PIM) in Germany's elderly as indicated by the PRISCUS list. An analysis based on regional claims data. Pharmacoepidemiol Drug Saf 2013;22:719-27.

21 Sönnerstam E, Sjölander M, Gustafsson M. An evaluation of the prevalence of potentially inappropriate medications in older people with cognitive impairment living in Northern Sweden using the EU(7)PIM list. Eur J Clin Pharmacol 2017;73:735-42.

22 Schäfer I, Hansen H, Schön G, et al. The influence of age, gender and socio-economic status on multimorbidity patterns in primary care. first results from the multicare cohort study. BMC Health Serv Res 2012;12:89.

23 Schäfer I, Hansen H, Schön G, et al. The German MultiCare-study: Patterns of multimorbidity in primary health care - protocol of a prospective cohort study. BMC Health Serv Res 2009;9:145.

24 DIMDI - ATC/DDD Anatomisch-therapeutisch-chemische Klassifikation mit definierten Tagesdosen. Available: http://www. dimdi.de/static/de/klassi/atcddd/index.htm

25 Van der Elst W, Dekker S, Hurks P, et al. The letter digit substitution test: demographic influences and regression-based normative data for school-aged children. Arch Clin Neuropsychol 2012;27:433-9.

26 Salthouse TA. The processing-speed theory of adult age differences in cognition. Psychol Rev 1996;103:403-28.

27 SSOAR. Educational reform in France, West-Germany and the United Kingdom: updating the CASMIN educational classification, 1999. Available: https://www.ssoar.info/ssoar/handle/document/20816

28 Grina D, Briedis V. The use of potentially inappropriate medications among the Lithuanian elderly according to Beers and EU(7)-PIM list a nationwide cross-sectional study on reimbursement claims data. $J$ Clin Pharm Ther 2017;42:195-200.

29 Wauters M, Elseviers M, Azermai M, et al. Availability and actual use in the Belgian market of potentially inappropriate medications (PIMs) from the EU(7)-PIM list. Eur J Clin Pharmacol 2016;72:243-5.

30 Wickop B, Härterich S, Sommer C, et al. Potentially inappropriate medication use in Multimorbid elderly inpatients: differences between the FORTA, PRISCUS and STOPP ratings. Drugs Real World Outcomes 2016;3:317-25.

31 Endres HG, Kaufmann-Kolle P, Knopf H, et al. Welche Faktoren begünstigen die Anwendung potenziell ungeeigneter Medikamente bei älteren Menschen? Bundesgesundheitsblatt Gesundheitsforschung Gesundheitsschutz 2018;61:40-51.

32 Vieira ER, Palmer RC, Chaves PHM. Prevention of falls in older people living in the community. BMJ 2016;353:i1419.

33 Kirchhof P, Benussi S, Kotecha D, et al. 2016 ESC guidelines for the management of atrial fibrillation developed in collaboration with EACTS. Eur Heart J 2016;37:2893-962.

34 Novaes PH, da Cruz DT, Lucchetti ALG, et al. Comparison of four criteria for potentially inappropriate medications in Brazilian community-dwelling older adults. Geriatr Gerontol Int 2017;17:1628-35.

35 Tommelein E, Mehuys E, Petrovic M, et al. Potentially inappropriate prescribing in community-dwelling older people across Europe: a systematic literature review. Eur J Clin Pharmacol 2015;71:1415-27.

36 Díez-Manglano J, Giménez-López M, Garcés-Horna V, et al. Excessive polypharmacy and survival in polypathological patients. Eur J Clin Pharmacol 2015;71:733-9.

37 Van der Elst W, Van Boxtel MPJ, Van Breukelen GJP, et al. Detecting the significance of changes in performance on the Stroop ColorWord test, Rey's verbal learning test, and the letter digit substitution test: the regression-based change approach. J Int Neuropsychol Soc 2008;14:71-80.

38 Maclagan LC, Maxwell CJ, Gandhi S, et al. Frailty and potentially inappropriate medication use at nursing home transition. J Am Geriatr Soc 2017;65:2205-12.

39 Wehling $\mathrm{M}$, Burkhardt $\mathrm{H}$, Kuhn-Thiel A, et al. VALFORTA: a randomised trial to validate the FORTA (fit fOR the aged) classification. Age Ageing 2016;45:262-7. 